\title{
Influence de la température sur les propriétés mécaniques et l'endommagement d'un composite carbone-thermoplastique
}

\author{
Xavier Gabrion, Vincent Placet, Lamine Boubakar \\ Institut FEMTO-ST - Département Mécanique Appliquée - UMR CNRS 6174 \\ 24 rue de l'épitaphe, F-25000 Besançon \\ xavier.gabrion@edu.univ-fcomte.fr \\ \{vincent.placet,lamine.boubakar\}@univ-fcomte.fr
}

RÉSUMÉ. Cet article propose une caractérisation thermomécanique d'un matériau composite à matrice organique thermoplastique thermostable renforcé par fibres de carbone. Les caractéristiques thermiques ainsi que les propriétés mécaniques sont étudiées sur une gamme de température s'étendant de l'ambiante à $200^{\circ} \mathrm{C}$. Un suivi de l'endommagement sous sollicitation thermomécanique est réalisé avec une technique de contrôle non destructif: l'émission acoustique. Cet article met en évidence l'influence de la température sur la résistance ultime et le mode de rupture du matériau sous sollicitations mécaniques dans le sens des fibres. Ces variations sont attribuées à la modification des forces d'adhésion à l'interface fibre/matrice. La ténacité à la rupture interlaminaire est également largement affectée par la température. Le taux de restitution d'énergie critique en mode $1\left(G_{I c}\right)$ diminue d'environ $60 \%$ entre l'ambiante et $110^{\circ} \mathrm{C}$. Ce travail montre enfin que la signature acoustique des différents modes d'endommagement est sensiblement affectée par la température.

ABSTRACT. This paper investigates the thermomechanical behavior of carbon fibre reinforced high performance thermoplastic composite. The thermal characteristics and mechanical properties are determined over a temperature range extending from ambient to $200^{\circ} \mathrm{C}$. Damage under thermomechanical loading is studied with a non destructive technique: Acoustic emission. This paper shows the influence of temperature on the specimen tensile breakage and ultimate strength in the fibre direction. This change is attributed to a modification of the adhesion forces at the fibre/matrix interface. The interlaminar fracture toughness is also shown to be influenced by temperature. The strain energy release rate determined in mode 1 delamination $\left(G_{I c}\right)$ decreases of approximately $60 \%$ from ambient temperature to $100^{\circ} \mathrm{C}$. This work also points out that acoustic signature of the different damage modes is significantly affected by temperature.

MOTS CLÉS: composite haute performance, matrice thermoplastique thermostable, haute température, émission acoustique.

KEYWORDS: high performance, thermostable thermoplastic matrix, composite, high temperature, acoustic emission. 


\section{Extended Abstract}

High-performance thermoplastic resins represent a promising alternative to thermosetting resins in advanced composite applications. The aim of this work is to accurately characterise the thermomechanical behaviour of carbon fibre reinforced thermostable thermoplastic polymer (Polyimide). Indeed, this type of composite is supposed to maintain its mechanical properties up to $200^{\circ} \mathrm{C}$. Many applications are expected in advanced engineering fields for this material such as pressure vessels, transportation systems, speed rotors, flywheels and so on. In these structures, the reinforcement is mainly oriented along the hoop axis. Filament winding is the most suitable manufacturing process for these products. In comparisons to thermosettings, thermoplastic composite offers possibility to produce structure in one shot by fibre placement and welding.

The main objective of this work is to quantify the effect of temperature on some physical and mechanical properties of the composite and to determine its suitable thermomechanical operating range. In this paper, material damage is also characterized using acoustic emission according to the temperature level.

Thermal expansion coefficient and thermal degradation kinetics are determined using various tests. Mass loss is achieved to be approximately $1 \%$ at a temperature of $450^{\circ} \mathrm{C}$ and the expansion coefficients are evaluated to $-1,7.10^{-6} /{ }^{\circ} \mathrm{K}$ in the fibre direction and $30.10^{-6} /{ }^{\circ} \mathrm{K}$ in the transverse direction. The mechanical properties are investigated on a temperature range from $-50^{\circ} \mathrm{C}$ to $200^{\circ}$ The matrix strength and rigidity are almost constant on this temperature range. If the strength is found to be approximately the same at room temperature and at $200^{\circ} \mathrm{C}$, a significant decrease of approximately $18 \%$ is measured at $110^{\circ} \mathrm{C}$. Interlaminar fracture toughness is also influenced by temperature. The strain energy release rate decreases of approximately $60 \%$ between room temperature and $100^{\circ} \mathrm{C}$. The viscoelastic properties of the material are also investigated using Dynamic Mechanical Analysis. The glass transition temperature is localised at a temperature of $250^{\circ} \mathrm{C}$. This work also demonstrates that the acoustic signature associated to the different damage modes are significantly affected by temperature.

\section{Introduction}

Les matériaux composites à matrice organique et fibres de carbone présentent des caractéristiques mécaniques élevées alliées à une faible densité. L'utilisation de ces matériaux dans les industries du transport représente un enjeu important dans la perspective d'un allègement des structures, et donc d'une réduction des coûts d'exploitation des véhicules et d'une diminution des émanations de gaz à effet de serre. Les matériaux composites à matrice thermodurcissable, très répandus à l'heure actuelle, en particulier dans le secteur aéronautique, présentent cependant quelques aspects néfastes face à cet argumentaire. Les matériaux composites à base de résine thermodurcissable ne sont, effectivement, pas recyclables et réparables uniquement 
par ajout d'un patch. Les pré-imprégnés à matrice thermodurcissable nécessitent, en sus, un stockage à température frigorifique. De plus, ces matériaux tolèrent des températures d'utilisation relativement faibles ne dépassant pas $120^{\circ} \mathrm{C}$ (exceptés pour quelques résines thermodurcissables thermostables utilisées dans des applications de niches) et peuvent contenir des substances cancérigènes (cf. Mc Connell, 2009). Dans ce contexte, l'utilisation de matériaux composites à matrice thermoplastique thermostable (TPTS) semble offrir une alternative interessante pour la réalisation de structures à hautes performances thermomécaniques. Les matrices TPTS, telles que le PEEK, le PPS, le PEI ont reçu une grande attention au sein de la communauté scientifique durant ces dernières décennies. Ces résines TPTS peuvent être réchauffées ou refondues offrant ainsi la possibilité de les façonner de manière non définitive, de les recycler, de les réparer ou de les souder (cf. Nicodeau, 2005 ; Ageorges et al., 2001). Leur stabilité thermique et leurs propriétés mécaniques intéressantes permettent d'envisager leur intégration dans des structures en zones chaudes, pouvant atteindre et dépasser $200^{\circ} \mathrm{C}$, actuellement réservées aux matériaux conventionnels, tels que les métalliques et céramiques (cf. Fleeman, 2006 ; Gao et al., 2001 ; Kim et al., 2004 ; Mahieux et al., 2002). Parmi les applications visées, certaines concernent des structures tubulaires ou annulaires dédiées au transport ou stockage de fluides, ou à la réalisation de rotors de moteur électrique ou de volants d'inertie. L'enroulement filamentaire est le procédé de fabrication le plus adapté à ces géométries, en plus de permettre une orientation aisée du renfort. Les propriétés des thermoplastiques donnent la possibilité de réaliser des pièces en «one shot », par placement et soudage de fibres (voir Nicodeau, 2005 ; Starnes et al., 2001), avec un gain de temps et d'énergie conséquent par rapport aux techniques traditionnelles d'enroulement filamentaire avec une résine thermodurcissable, nécessitant une phase de polymérisation. Ces structures, lorsqu'elles sont réalisées en composite, présentent un renfort orienté principalement dans la direction circonférentielle afin de résister aux fortes contraintes générées en service dans cette même direction. Bien que dominant dans cette direction, le niveau des contraintes dans les autres directions matérielles est loin d'être négligeable dans la plupart des applications citées précédemment, et en plus d'être multiaxiales, ces contraintes sont souvent appliquées de manière cyclique et combinées à un cyclage thermique. La connaissance de la tolérance et de la durabilité des matériaux est donc cruciale dans ces applications. Cependant, peu d'études de caractérisation mécanique ont été réalisées sur le CMO à matrice TPTS. Ce travail a pour objectif d'étudier et de quantifier l'effet de la température sur les propriétés physiques et mécaniques et sur les modes d'endommagement et de rupture d'un composite à fibres de carbone et matrice polyimide thermoplastique. Une attention particulière est portée sur l'évaluation de l'effet de la température sur les signatures acoustiques des différents modes d'endommagement. 


\section{Matériel et méthodes}

\subsection{Matériau}

Le matériau de l'étude est un composite unidirectionnel (UD). Il est distribué sous forme de ruban, composé de 4 mèches de 12000 fibres de carbone T700S imprégnées de résine polyimide thermoplastique. Le taux volumique de fibres est de $50 \%$. La largeur du ruban est de $13 \pm 0,5 \mathrm{~mm}$ et son épaisseur de $0.29 \pm 0,02 \mathrm{~mm}$. Des stratifiés UD $\left[0^{\circ}\right]_{10}$ ont été réalisés par compression à chaud à une température de $400{ }^{\circ} \mathrm{C}$ et sous une pression de 6 bars. La vitesse de chauffage est de $2{ }^{\circ} \mathrm{C} / \mathrm{min}$, la vitesse de refroidissement d'environ $1,5^{\circ} \mathrm{C} / \mathrm{min}$. Pour les éprouvettes destinées aux essais de délaminage, un film anti-adhésif en téflon a été inséré entre la $5^{\mathrm{e}}$ et $6^{\mathrm{e}}$ couche ; il a pour rôle l'amorçage de la fissure.

\subsection{Caractérisation thermomécanique}

\subsubsection{Expansion thermique}

Les coefficients d'expansion thermique (CET) ont été déterminés dans les directions transversale et longitudinale sur le stratifié à l'aide d'un dilatomètre Netzsch DIL $402 \mathrm{C}$ en atmosphère oxydante entre 20 et $400{ }^{\circ} \mathrm{C}$ avec une vitesse de chauffage de $2{ }^{\circ} \mathrm{C} / \mathrm{min}$. Trois échantillons ont été testés dans chaque direction matérielle avec des dimensions de $8.5 * 5 * 2,2 \mathrm{~mm}^{3}$. Les CET ont été déterminés par régression linéaire à partir de la courbe température/déformation sur la plage de température $50-200^{\circ} \mathrm{C}$.

\subsubsection{Dégradation thermique}

La dégradation du matériau par la température a été investiguée en atmosphères oxydante et inerte. Un analyseur thermogravimétrique (ATG) SETARAM a servi à étudier la dégradation thermique du matériau sous argon. La perte de masse a été mesurée entre 20 et $700{ }^{\circ} \mathrm{C}$ avec une vitesse de chauffage monotone de $5^{\circ} \mathrm{C} / \mathrm{min}$. Les mesures ont été réalisées sur 3 échantillons d'une masse de $60 \mathrm{mg}$. Les essais en atmosphère oxydante ont été réalisés suivant la norme ASTM D 2584-02 dans un four. Les mesures ont été réalisées au niveau de paliers isothermes pour différents temps d'exposition. La vitesse de chauffage est de $4{ }^{\circ} \mathrm{C} / \mathrm{min}$ pour atteindre les différents paliers de température étudiés. Les essais ont été réalisés sur des échantillons de ruban et de stratifié.

\subsubsection{Propriétés mécaniques en traction}

Les propriétés mécaniques en traction du ruban, module d'Young, contrainte à rupture et déformation à rupture, ont été déterminées dans la direction longitudinale (parallèle aux fibres). Les essais ont été réalisés à l'aide d'une machine électromécanique (Instron 6025) de capacité $\pm 100 \mathrm{kN}$, conformément à la norme 
ASTM D 3039-00. Les essais ont été réalisés à cinq températures : -50, -20, 23, 110 et $200{ }^{\circ} \mathrm{C}$ à une vitesse de chargement de $1 \mathrm{~mm} / \mathrm{min}$. Des talons métalliques de $50 * 25 \mathrm{~mm}^{2}$ ont été collés aux extrémités des éprouvettes, de dimensions $250 * 13 * 0.29 \mathrm{~mm}^{3}$ à l'aide d'une colle bi-composant Duralco 4703. Les déformations ont été mesurées à l'aide d'un extensomètre laser (EIR LE05) doté d'une précision de mesure du déplacement de l'ordre du micron. La zone utile pour la mesure des déformations est de $50 \mathrm{~mm}$. Le module d'Young longitudinal a été identifié par régression linéaire à partir de la courbe contrainte/déformation entre 0 et $0,4 \%$ de déformation.

\subsubsection{Propriétés viscoélastiques}

Le comportement mécanique dans la direction transversale a été étudié sur le stratifié, les dimensions du ruban ne permettant pas de prélever des éprouvettes. Les propriétés viscoélastiques ont été déterminées à l'aide d'un DMA (Dynamics Mechanical Analysis) Bose ElectroForce entre 30 et $275^{\circ} \mathrm{C}$ au niveau de paliers isothermes espacés de $5{ }^{\circ} \mathrm{C}$. La vitesse de chauffage était de $3{ }^{\circ} \mathrm{C} / \mathrm{min}$ entre chaque palier et un délai de 2 minutes a été respecté à chaque palier avant d'effectuer les mesures de façon à garantir l'homogénéité de la température au sein de l'éprouvette. Le signal imposé était sinusoïdal de valeur moyenne $5 \mathrm{~N}$ et d'amplitude crête-crête $8 \mathrm{~N}$. Ces essais ont permis de déterminer les modules de stockage E', de perte E' ainsi que le facteur de perte $(\tan \delta$ ). La température de transition vitreuse Tg est identifiée à partir du pic du module de perte.

\subsubsection{Ténacité à la rupture interlaminaire en mode 1}

La ténacité à la rupture interlaminaire a également été déterminée en mode 1 à différentes températures $\left(23,100,150\right.$ et $\left.200^{\circ} \mathrm{C}\right)$. Les essais ont été réalisés à l'aide d'une machine électromécanique dotée d'une cellule de force $\pm 500 \mathrm{~N}$ avec une vitesse de déplacement imposé à $1 \mathrm{~mm} / \mathrm{min}$. La dimension des éprouvettes est de $150 * 12 * 2,5 \mathrm{~mm}^{3}$. Elles ont été découpées à la scie diamantée, les côtés étant peints en blanc et marqués tous les $5 \mathrm{~mm}$ de façon à suivre la croissance de fissure. Celleci a été suivie par une caméra optique Kodak megaplus. La détermination expérimentale du taux de restitution d'énergie critique $\left(G_{I C}\right)$ est réalisée suivant la méthode décrite dans la norme NF ISO 15024, basée sur l'utilisation d'éprouvette DCB (Double Cantilever Beam). $G_{I c}$ est estimé en relevant au cours de l'essai la longueur de délaminage $(a)$, la force exercée $(P)$ ainsi que le déplacement de la traverse $(\delta)$. Notons $(b)$ la largeur de l'éprouvette et $C=\delta / P$ la complaisance. Dans le cas d'un essai à déplacement imposé $G_{I C}$ s'écrit sous la forme :

$$
G_{I c}=\frac{1}{2 b} P^{2} \frac{\partial C}{\partial a}
$$

La méthode utilisée pour le calcul du terme $\partial C / \partial a$ est la méthode MCC (Modified Compliance Calibration). Elle donne l'expression de ce terme en fonction de la 
charge, de la complaisance, des dimensions de l'éprouvette $b$ et $e$ (épaisseur) et de facteurs correctifs $F$ et $N$ (2). Le coefficient $m$ est donné par la pente de la droite $(b C / N)^{1 / 3}$ en fonction de la longueur de délaminage normalisée par l'épaisseur $a / 2 e$.

$$
G_{I c}=\frac{3 m}{2(2 e)}\left(\frac{P}{b}\right)^{2}\left(\frac{b C}{N}\right)^{2 / 3} F
$$

\subsubsection{Suivi de l'endommagement}

L'activité acoustique générée lors de l'endommagement du matériau sous les différentes sollicitations mécaniques a été enregistrée grâce à un système Physical Acoustics Coproration (PAC) équipé de deux capteurs micro 80 pré-amplifiés à $40 \mathrm{~dB}$. Les paramètres d'acquisition sont les suivants : seuil $=40 \mathrm{~dB}$, PDT $=20 \mu \mathrm{s}$, $\mathrm{HDT}=120 \mu \mathrm{s}, \mathrm{HLT}=500 \mu \mathrm{s}$. En traction longitudinale, deux capteurs ont été placés aux extrémités de l'éprouvette sur les talons métalliques. En délaminage, un seul capteur a été positionné à l'extrémité de l'éprouvette.

\section{Résultats et discussions}

La figure 1 représente les pertes de masse provoquées par la dégradation thermique du matériau sous atmosphères inerte et oxydante. Une diminution de la masse de $1 \%$ est observée à $500^{\circ} \mathrm{C}$ et seulement $13 \%$ à $700{ }^{\circ} \mathrm{C}$ en atmosphère inerte (argon). En revanche, la figure montre clairement la forte influence de la nature de l'atmosphère sur la cinétique de dégradation. En effet, des pertes de masse de $2 \%$ et $41 \%$ sont observées à respectivement $450{ }^{\circ} \mathrm{C}$ et $550{ }^{\circ} \mathrm{C}$ après $1 \mathrm{~h}$ d'exposition en atmosphère oxydante. Ce matériau présente donc une bonne résistance à la dégradation thermique jusqu'à $450^{\circ} \mathrm{C}$. Ce résultat permet de cartographier les zones de durée/température d'exposition tolérables pour le matériau, données essentielles pour l'optimisation des procédés de fabrication.

Les essais dilatométriques réalisés ont révélé un comportement fortement anisotrope entre les directions longitudinale et transversale. Des exemples de courbes dilatométriques pour chaque direction matérielle sont donnés en figure 2a. Les coefficients d'expansion thermique identifiés sont en moyenne $-1,7.10^{-6} \mathrm{~K}^{-1}$ et $30.10^{-6} \mathrm{~K}^{-1}$ pour les directions longitudinale et transversale. La différence de signes entre les directions matérielles principales est à souligner, il est en effet bien connu que celle-ci peut conduire à une ondulation de la fibre dans la matrice lors de la phase de refroidissement au cours de la fabrication des structures. Ce phénomène, bien connu pour les résines thermodurcissables (cf. Parlevliet et al., 2007 ; Placet et al., 2011), pourrait être minimisé dans le cas des résines TPTS, les procédés de fabrication par soudage impliquant des zones de chauffage très localisées et de très courtes durées. Les courbes dilatométriques permettent de faire apparaître également deux transitions. La première à $230{ }^{\circ} \mathrm{C}$ correspond à la transition vitreuse, la seconde à $370{ }^{\circ} \mathrm{C}$ quant à elle correspond à la température de fusion de ce thermoplastique 
semi-cristallin. La température de transition vitreuse a également été identifiée à l'aide des essais DMA.

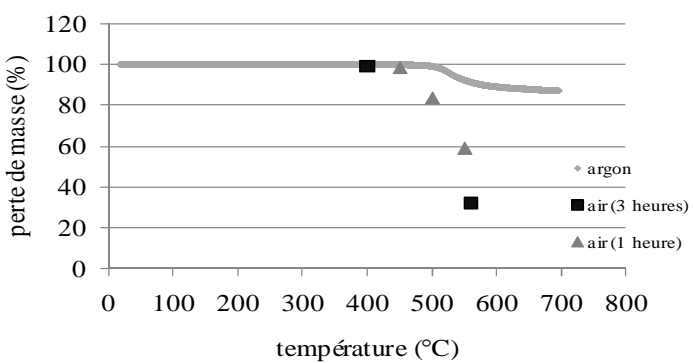

Figure 1. Évolution de la perte de masse en fonction de la température en atmosphère inerte et oxydante
Trait : vitesse de chauffage constante en atmosphère inerte, points : paliers isothermes en atmosphère oxydante

La $T g$ est localisée à $260{ }^{\circ} \mathrm{C}$ pour une fréquence de sollicitation de $1 \mathrm{~Hz}$. La différence enregistrée entre les essais dilatométriques et harmoniques sur la valeur de $T g$ est à attribuer à la vitesse de sollicitation. L'évolution de la température de transition vitreuse en fonction de la fréquence de sollicitation est donnée en figure $2 \mathrm{~b}$.On note une variation de la $\mathrm{Tg}$ d'environ $5{ }^{\circ} \mathrm{C}$ par décade de fréquence.
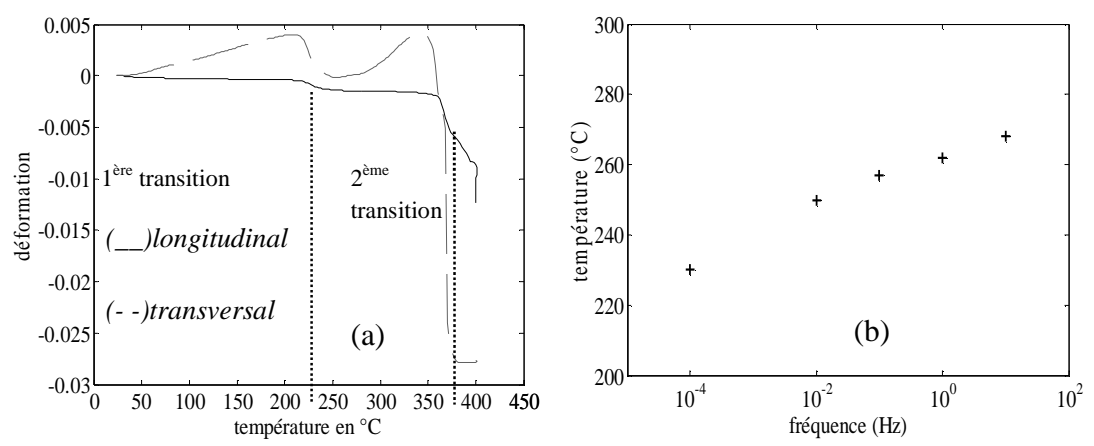

Figure 2. (a) Coefficient d'expansion thermique. (b) Évolution de la température de transition vitreuse en fonction de la fréquence de sollicitation

Les courbes du module de stockage E' et du module de perte E' en fonction de la température sont données en figure 3. Le module de stockage apparaît comme relativement stable jusqu'à $240^{\circ} \mathrm{C}$ avec une diminution d'environ $8 \%$ seulement jusqu'à cette température. Une forte diminution, d'un facteur proche de 5 , est alors observée au niveau de la $T g$. Le module de perte est quant à lui stable jusqu'à $200{ }^{\circ} \mathrm{C}$, température à partir de laquelle il croît de façon significative jusqu'à la Tg. 
Une légère variation de $\mathrm{E}$ ', peut-être due à une relaxation secondaire, est observée à $120^{\circ} \mathrm{C}$. Néanmoins ces caractéristiques mécaniques dans la direction transversale confirment la bonne stabilité de la rigidité de ce thermoplastique jusqu'à $250^{\circ} \mathrm{C}$.

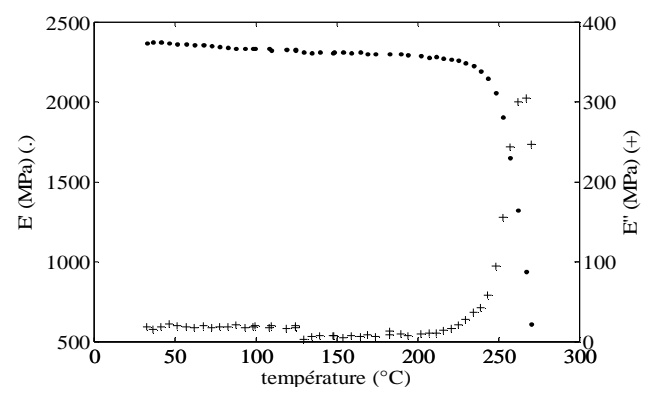

Figure 3. Évolution du module de stockage E' (.) et module de perte E', (+) en fonction de la température à une fréquence de sollicitation de $1 \mathrm{~Hz}$

Tableau 1. Propriétés mécaniques et type de rupture observé dans la direction longitudinale à différentes températures

\begin{tabular}{|c|c|c|c|c|}
\hline $\begin{array}{c}\text { Température } \\
\text { des essais }\end{array}$ & $\begin{array}{c}\text { Nombre } \\
\text { d'éprouvettes }\end{array}$ & $\begin{array}{c}\text { Module } \\
\text { d'Young }(\mathrm{GPa})\end{array}$ & $\begin{array}{c}\text { Contrainte à } \\
\text { rupture (MPa) }\end{array}$ & $\begin{array}{c}\text { Type de rupture } \\
\text { cascade/brosse (\%) }\end{array}$ \\
\hline$-50^{\circ} \mathrm{C}$ & 3 & $\mathrm{nc}$ & $1341 \pm 154$ & $100 / 0$ \\
\hline$-20^{\circ} \mathrm{C}$ & 3 & $\mathrm{nc}$ & $1667 \pm 528$ & $100 / 0$ \\
\hline $23{ }^{\circ} \mathrm{C}$ & 8 & $111 \pm 8,5$ & $1775 \pm 275$ & $35 / 65$ \\
\hline $110^{\circ} \mathrm{C}$ & 8 & $\mathrm{nc}$ & $1500 \pm 300$ & $100 / 0$ \\
\hline $200^{\circ} \mathrm{C}$ & 6 & $\mathrm{nc}$ & $1730 \pm 320$ & $67 / 33$ \\
\hline
\end{tabular}

Les propriétés mécaniques déterminées dans la direction longitudinale en traction sont synthétisées dans le tableau 1. À température ambiante, le module d'Young longitudinal est d'environ $110 \mathrm{GPa}$ et la contrainte à rupture moyenne de $1775 \mathrm{MPa}$. Il est possible de constater que la température affecte la résistance du matériau, avec une diminution de $18 \%$ en moyenne entre l'ambiante et $110{ }^{\circ} \mathrm{C}$. Cette tendance s'inverse à partir de cette température, pour retrouver une contrainte à rupture moyenne proche de celle de l'ambiante à $200^{\circ} \mathrm{C}$. On peut également noter une diminution de la contrainte à rupture moyenne de $6 \%$ à $-20^{\circ} \mathrm{C}$ et de $24 \%$ à $-50{ }^{\circ} \mathrm{C}$ par rapport à la température ambiante. Cependant, les essais sont entachés d'une forte dispersion. Afin d'étudier plus précisément la tendance observée sur la contrainte à rupture moyenne, une distribution des valeurs de contraintes à rupture est tracée suivant une loi de Weibull. Cette méthode probabiliste est basée sur des hypothèses de 
mécanique de la rupture, ainsi que sur la propagation de défauts de taille critique conduisant à une rupture fragile du matériau. La probabilité de rupture s'exprime par :

$$
P_{f}=1-\exp \left[-\frac{V}{V_{0}}\left(\frac{\sigma_{r}}{\sigma_{0}}\right)^{m}\right]
$$

avec $P_{f}$ la probabilité de rupture, $\sigma_{r}$ la contrainte à rupture, $\sigma_{0}$ la contrainte caractéristique, $\mathrm{V}_{0}$ le volume de référence choisi arbitrairement, $\mathrm{V}$ le volume de l'éprouvette et $m$ le module de Weibull.

Les contraintes à rupture, classées en ordre croissant, sont affectées d'une probabilité de rupture définie par :

$$
P_{i}=(i-0.5) / n
$$

avec $n$ le nombre d'éprouvettes testées, $i$ la ième éprouvette. En traçant $\ln \sigma_{r}$ en fonction de $\ln (\ln (1 /(1-P i)))$, il est possible d'identifier le module de Weibull à partir d'une régression linéaire (cf. figures $4 a$ et $b$ ).

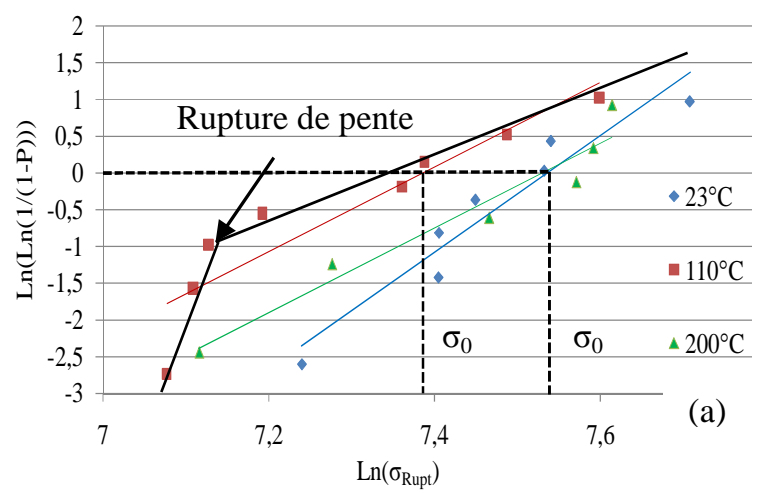

\begin{tabular}{|c|c|c|}
\hline $\mathrm{T}\left({ }^{\circ} \mathrm{C}\right)$ & $\sigma_{0} M P a$ & $\mathrm{~m}$ \\
\hline $23{ }^{\circ} \mathrm{C}$ & 1876 & 7,93 \\
\hline $110^{\circ} \mathrm{C}$ & 1616 & 5,75 \\
\hline $20{ }^{\circ} \mathrm{C}$ & 1866 & 5,76 \\
\hline
\end{tabular}

Figure 4. (a) Distribution des contraintes à rupture suivant une loi de Weibull pour les essais de traction dans la direction longitudinale à 23,110 et $200{ }^{\circ} \mathrm{C}$

(b) Contraintes caractéristiques et module de Weibull aux différentes températures

Les valeurs des contraintes caractéristiques ainsi que le module de Weibull sont répertoriés dans la figure $4 \mathrm{~b}$. Les modules de Weibull sont à considérer avec prudence étant donné le faible nombre d'éprouvettes testées (10) et la forte nonlinéarité de la courbe obtenue à $110^{\circ} \mathrm{C}$. L'ordre de grandeur est néanmoins en accord avec la valeur de 5.62 mesurée par Blassiau (2005) sur la fibre de carbone. Cette forte non-linéarité semble suggérer l'existence de deux mécanismes de rupture basés sur des familles de défauts ou de tailles de défauts différentes. Néanmoins, les contraintes caractéristiques suivent la même tendance que celle observée à partir des 
contraintes moyennes. La chute de la résistance à une température de $110^{\circ} \mathrm{C}$ est statistiquement significative.

De plus, les comportements à rupture à $-20^{\circ} \mathrm{C},-50{ }^{\circ} \mathrm{C}$ et $110^{\circ} \mathrm{C}$ sont différents de ceux observés à l'ambiante et à $200^{\circ} \mathrm{C}$ (cf. tableau 1). Pour les trois premières températures toutes les éprouvettes présentent une rupture en cascade, c'est-à-dire par propagation de la rupture des fibres à travers une même section de ruban, avec peu ou pas de scission longitudinale (cf. figure 5a (2)). Pour éviter toute confusion au niveau du vocabulaire, la scission est entendue ici comme la propagation d'une fissure matricielle le long des fibres, conduisant à la division d'une éprouvette en deux morceaux. Pour les éprouvettes testées à température ambiante et à $200{ }^{\circ} \mathrm{C}$, deux comportements à rupture sont observés, le type «cascade » décrit précédemment et le type «brosse», pour lequel les ruptures de fibres sont dispersées à toutes les altitudes de l'éprouvette avec une succession des phénomènes de rupture de fibres et scission longitudinale (cf. figure $5 \mathrm{a}(1)$ ).

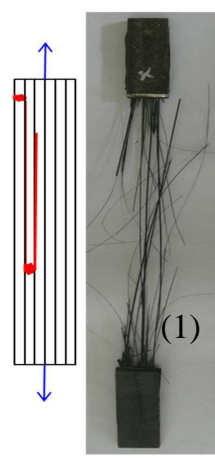

(a)

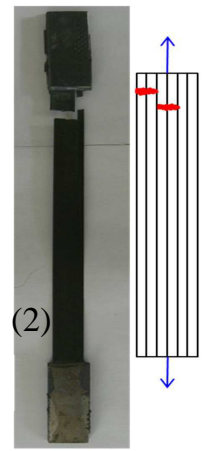

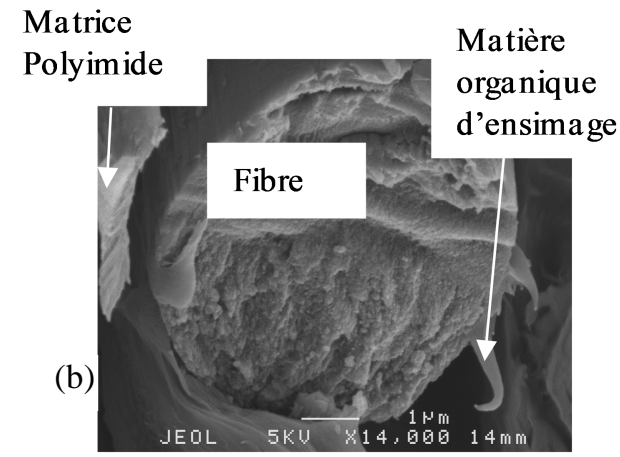

Figure 5. (a) Faciès de rupture observés en traction longitudinale, rupture en brosse (1), rupture en cascade (2). (b) Cliché MEB d'une fibre de carbone mettant en évidence la fine couche de matière organique utilisée pour l'ensimage entre la fibre et la matrice polyimide

Ces comportements à rupture sont largement étudiés dans la littérature (voir Van den Heuvel et al., 1996 ; Holmes et al., 2002) et sont attribués pour le type « brosse » à une matrice à comportement fragile et une interface fibre/matrice relativement faible, et une interface forte dans le cas des ruptures en cascade. L'augmentation du pourcentage de rupture de type « cascade » à $110^{\circ} \mathrm{C}$ et $200^{\circ} \mathrm{C}$ reflète certainement une modification de l'adhésion à l'interface fibre/matrice, peut-être provoquée par la transition vitreuse de la matière organique utilisée pour l'ensimage des fibres de carbone (cf. figure 5b). Cette modification de comportement à rupture à partir de $110^{\circ} \mathrm{C}$ pourrait expliquer la non-linéarité de la courbe $\ln (\ln (1 /(1-P i)))$ en fonction de $\ln \sigma_{r}$ (cf. figure $4 \mathrm{a}$ ). La transition secondaire localisée lors des essais 
DMA pourrait être due à cette relaxation à l'interface fibre/matrice. Malgré cette variation notable à $110^{\circ} \mathrm{C}$, les performances mécaniques du ruban restent élevées jusqu'à $200^{\circ} \mathrm{C}$ ainsi que dans les températures négatives. Au-delà de cette température, la $T g$ de la matrice et la dégradation de l'adhésif permettant la fixation des talons rendent les essais de traction inexploitables. De même, les mesures de déformation (et par ce fait du module d'Young) n'ont pas été présentées, les mouvements convectifs de l'air chaud à l'intérieur de l'enceinte thermique perturbant la propagation du faisceau laser de l'extensomètre. Un effort est à fournir au niveau de la mesure des déformations afin d'accéder à des données fiables.

Ces essais simples, favorisant un ou deux mécanismes d'endommagement (rupture de fibres et scission), ont constitué un support privilégié pour déterminer la signature acoustique des modes d'endommagement. Effectivement, ce travail d'identification des signatures acoustiques est particulièrement important dans l'optique d'un travail futur, consistant à dissocier et identifier des mécanismes d'endommagement à partir de leur signature acoustique à l'aide d'outils de reconnaissance supervisés dans le cas d'essais de fatigue multiaxiale, pour lesquels de nombreux mécanismes d'endommagement peuvent apparaître successivement ou simultanément. D'une manière assez générale, pour les composites à matrice organique renforcés de fibres longues, la fissuration matricielle se traduit par des événements de faible amplitude $(\approx 40$ à $60 \mathrm{~dB})$ et de courte durée, le délaminage et les phénomènes de décohésion fibre/matrice par des amplitudes intermédiaires $(\approx 60$ à $80 \mathrm{~dB})$ et des longues durées et la rupture de fibres par des fortes amplitudes $(\approx 80$ à $100 \mathrm{~dB}$ ) et des longues durées (cf. Barré et al., 1994 ; Ely et al., 1995). Pour ces essais de traction sur ruban dans le sens des fibres, la distribution des salves d'EA en fonction de leur amplitude est présentée pour des températures de $23{ }^{\circ} \mathrm{C}$ et $110^{\circ} \mathrm{C}$, respectivement en figures $6 \mathrm{a}$ et $6 \mathrm{~b}$. Les graphiques présentés correspondent aux résultats d'un seul essai. La répétabilité et la reproductibilité des lois de distribution ont été vérifiées pour les différents échantillons testés dans les mêmes conditions. La distribution des événements couvre toute la gamme d'amplitude (40 à $100 \mathrm{~dB})$. Notons une différence significative dans les fortes amplitudes (> $70 \mathrm{~dB})$, le nombre d'événement est plus important et surtout dans les très fortes amplitudes entre 90 et $98 \mathrm{~dB}$. Cette différence notable au niveau de la population des amplitudes intermédiaires est attribuée aux mécanismes de scission se produisant dans les ruptures de type «brosse». La distribution des salves en fonction de la durée est présentée en figures $6 \mathrm{c}$ et $6 \mathrm{~d}$. Malgré un nombre de salves plus faible à $110^{\circ} \mathrm{C}$ qu'à l'ambiante, la forme de la fonction de distribution est similaire avec des durées centrées autour d'une valeur médiane d'environ $100 \mu \mathrm{s}$. En revanche, la distribution des salves d'EA en fonction de leur énergie absolue (figures 6e et 6f) est fortement affectée par la température, avec une forte réduction de l'énergie absolue cumulée à $110^{\circ} \mathrm{C}$, traduisant le caractère beaucoup plus énergétique d'une rupture en « brosse » par rapport à une rupture en « cascade ».

Le comportement en délaminage a également été étudié à partir d'éprouvettes stratifiées. Ce mode d'endommagement est particulièrement important dans les 
structures tournantes (cf. Ratner et al., 2003). La courbe force/déplacement (cf. figure 7a) illustre le déroulement d'un essai de délaminage avec une première charge/décharge correspondant à l'amorçage de la fissure. Ensuite une charge de propagation sert à identifier les valeurs de $\mathrm{G}_{1 \mathrm{p}}$. La moyenne de cette propriété est faite pour les valeurs de déplacement de la traverse comprises entre 10 et $55 \mathrm{~mm}$. Les premiers points de $G_{I C}$ correspondent aux points d'initiation (où d'amorçage).
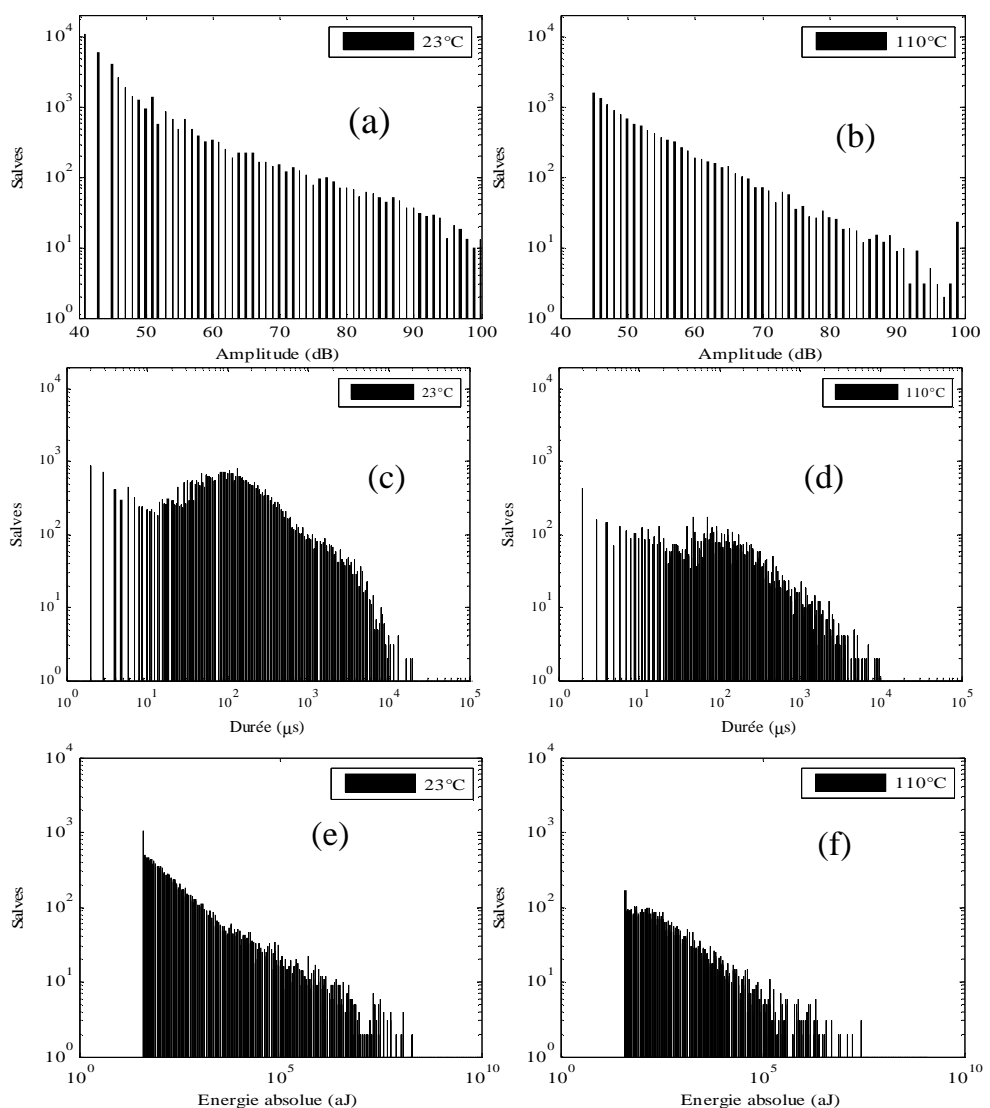

Figure 6. Distribution du nombre de salves en fonction de l'amplitude (a) et (b), de la durée (c) et (d) et de l'énergie absolue (e) et (f) en traction longitudinale pour respectivement $23^{\circ} \mathrm{C}$ et $110^{\circ} \mathrm{C}$

La figure $7 \mathrm{~b}$ présente les valeurs moyennes du taux de restitution d'énergie critique mesurées aux différentes températures de conditionnement des essais. Le $G_{I C}$ chute d'une valeur de $0,97 \mathrm{KJ} / \mathrm{m}^{2}$ à l'ambiante à $0,36 \mathrm{KJ} / \mathrm{m}^{2}$ à $100^{\circ} \mathrm{C}$, pour se stabiliser ensuite à cette valeur jusqu'à $200{ }^{\circ} \mathrm{C}$. Un constat opposé est fait par Kim et 
al. (2004) sur un composite FC/PEI où $G_{I c}$ augmente de $2,5 \mathrm{~kJ} / \mathrm{m}^{2}$ à $4 \mathrm{~kJ} / \mathrm{m}^{2}$ pour des températures variant de 25 à $130^{\circ} \mathrm{C}$. Ils attribuent cette augmentation à un fort ramollissement de la matrice permettant alors au phénomène de pontage de fibres de s'exprimer pleinement au cours de l'essai, le pontage conduisant à une augmentation de la résistance interlaminaire.
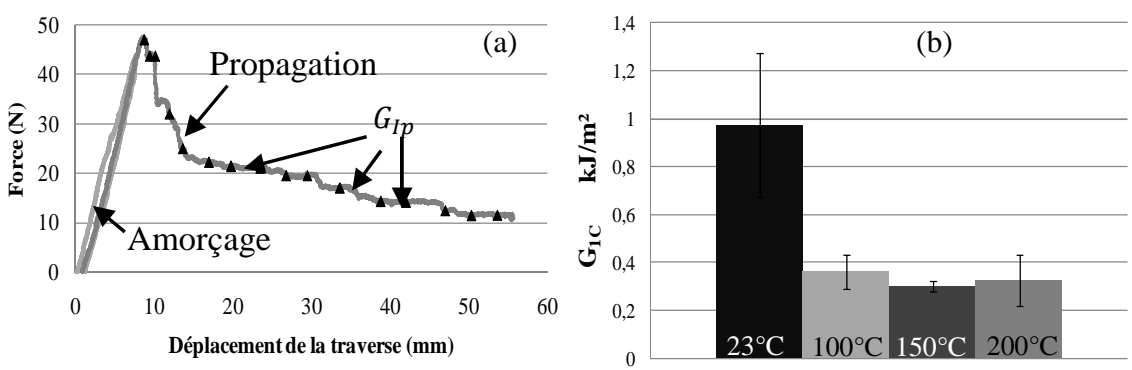

Figure 7. (a) Courbe force/déplacement de la traverse enregistrée au cours d'un essai de délaminage. (b) $G_{l c}$ en fonction de la température environnante

Dans notre cas, les essais DMA ont montré qu'aucun ramollissement majeur du polyimide ne se produit avant $230{ }^{\circ} \mathrm{C}$. Gao et al. (2001) montrent que la diminution de $G_{I c}$ est due à une augmentation du taux de cristallinité et donc de la ductilité de la matrice. Des mesures de taux de cristallinité, en cours sur les éprouvettes de délaminage, devraient permettre d'infirmer ou de confirmer cette hypothèse dans le cadre de cette étude. La signature acoustique des essais de délaminage a également été étudiée en fonction de la température. La figure $8 \mathrm{met}$ en évidence les différences enregistrées au niveau de la signature acoustique du délaminage en mode 1 à $23{ }^{\circ} \mathrm{C}$ et à $100{ }^{\circ} \mathrm{C}$. Là encore, bien que la gamme d'amplitude soit la même pour les deux températures, la forme de la loi de distribution change sensiblement. Comme pour les essais de traction, l'effet de la température est particulièrement marqué au niveau de l'énergie absolue des événements en accord avec la diminution de la ténacité aux températures les plus élevées.

Ces résultats expérimentaux permettent également de confirmer la différence majeure entre la rupture de fibres et le délaminage au niveau de la durée des salves. Celles-ci sont comprises pour ces deux types d'endommagement entre 0 et $10^{4} \mu \mathrm{s}$, mais la distribution est centrée sur des événements plus longs (1000 $\mu$ s) en délaminage (cf. figure $8 \mathrm{c}$ ) et beaucoup plus courts $(100 \mu \mathrm{s})$ pour les ruptures de fibres (cf. figure 6c). 

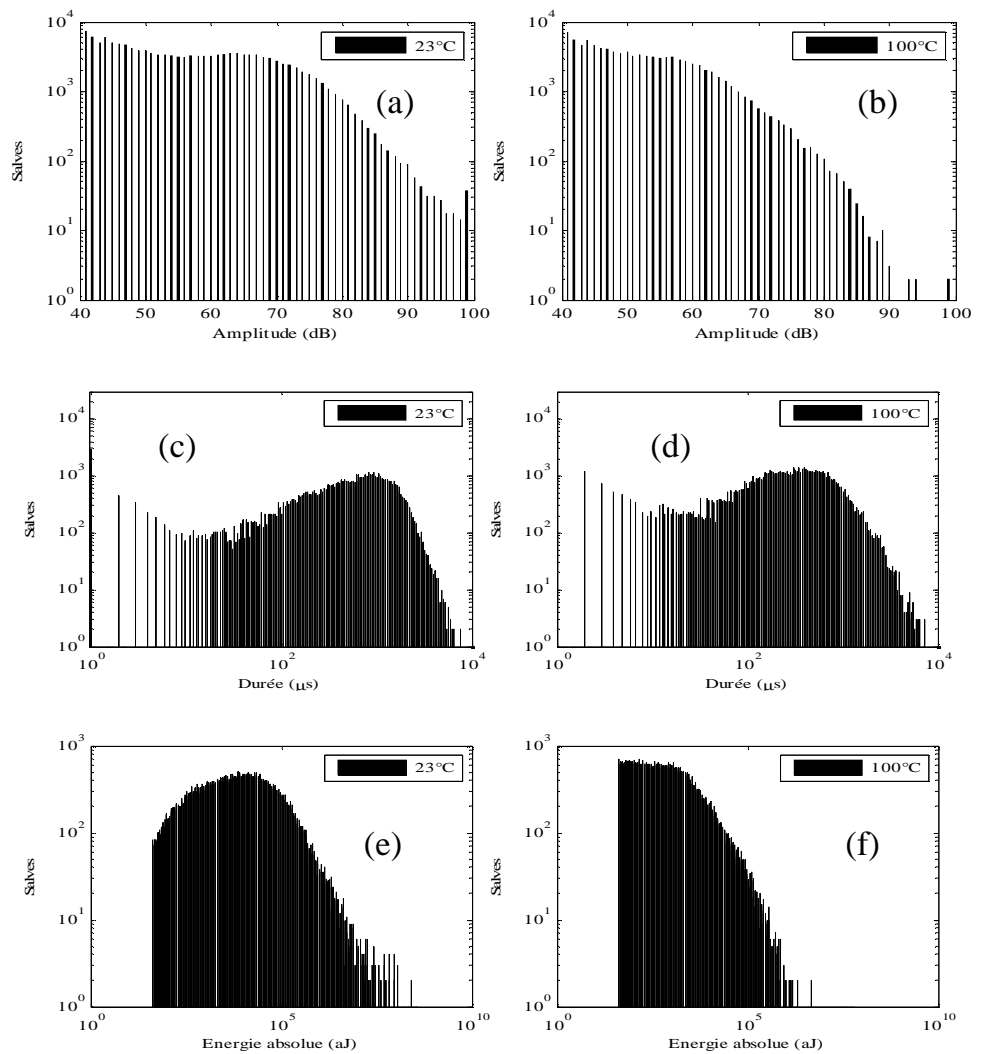

Figure 8. Nombre de salves en fonction de l'amplitude (a) et (b), de la durée (c) et $(d)$ et de l'énergie absolue (e) et (f) en délaminage pour $23{ }^{\circ} \mathrm{C}$ et $110^{\circ} \mathrm{C}$

\section{Conclusion}

Les performances thermomécaniques d'un matériau composite à fibres de carbone et matrice polymère polyimide ont été étudiées sur une large gamme de températures $\left(23\right.$ à $\left.200{ }^{\circ} \mathrm{C}\right)$. Les différents essais réalisés montrent une bonne stabilité de la rigidité de la matrice et de la résistance du composite sur la gamme de température visée. Une diminution de la résistance de l'ordre de $15 \%$ est néanmoins à prendre en compte pour les températures intermédiaires (aux environs de $110^{\circ} \mathrm{C}$ ). Le matériau offre une résistance thermique pour des courtes durées d'exposition jusqu'à des températures de $450-500{ }^{\circ} \mathrm{C}$ en atmosphère oxydante. Par contre, la ténacité à la rupture interlaminaire est fortement affectée par la température. Ce travail a également permis d'identifier les signatures acoustiques des différents modes d'endommagement et des modifications induites par la température. 


\section{Bibliographie}

Ageorges C., Ye Lin, Hou M. (2012). Advances in fusion bonding techniques for joining thermoplastic matrix composites: a rewiew. Composite part A, vol. 32, p. 839-857.

Barré S., Benzeggagh M.L. (1994). On the use of acoustic emission to investigate damage mechanisms in glass-fibre-reinforced polypropylene. Composites Science and Technology, vol. 52, p. 369-376.

Blassiau S. (2005). Modélisation des phénomènes microstructuraux au sein d'un composite unidirectionnel carbonelepoxy et prédiction de durée de vie : contrôle et qualification de réservoirs bobinés. Thèse en Sciences et Génie des Matériaux, ENSMP Paris.

D2584-02 (2002). Standard test method for ignition loss of cured reinforced resin, ASTM.

D3039-00 (2002). Standard test method for tensile properties of polymer matrix composite materials. ASTM.

Ely T.M., Hill E.v.K. (1995). Longitudinal splitting and fiber breakage characterization in graphite epoxy using acoustic emission data. Materials Evaluation, p. 288-294.

Fleeman E.L. (2006). Tactical missile design. Amer. Inst. of Aeronautics.

Gao S.L., Kim J.K. (2001). Cooling rate influences in carbon fibre/PEEK composites. Part II: interlaminar fracture toughness. Composites Part A, vol. 32, $n^{\circ}$ 6, p. 763-774.

Holmes G.A., McDonough W.G. (2002). E-Glass/DGEBA/m-PDA Model Composites: Time Dependent Failure in a Brittle Multi-Fiber Composite. $47^{\text {th }}$ International SAMPE Symposium and Exhibition.

Kim K.Y., Ye L. (2004). Interlaminar fracture toughness of CF/PEI composites at elevated temperatures: roles of matrix toughness and fibre/matrix adhesion. Composites Part A: Applied Science and Manufacturing, vol. 35, $\mathrm{n}^{\circ}$ 4, p. 477-487.

Mahieux C.A., Lehmann D., DesLigneris A. (2002). Experimental determination of the effects of industrial oil immersion on polymer-based composites. Polymer testing, vol. 21, $\mathrm{n}^{\circ} 7$, p. 751-756.

Mc Connell V.P. (2009). Resins for the hot zone, part 1: Polyimides. http://www.Composites world.com/articles/resins-for-the-hot-zone-part-i-polyimides.html

NF ISO 15024 (2002). Détermination de la ténacité à la rupture interlaminaire en mode $\mathrm{I}, G_{1 c}$, de matériaux composites à matrice polymère renforcés de fibres unidirectionnelles.

Nicodeau C. (2005). Modélisation du soudage en continu de composites à matrice thermoplastique. Thèse en Mécanique et Matériaux, ENSAM Paris.

Parlevliet P.P., Bersee HEN., Beukers A. (2007). Residual stresses in thermoplastic composites. Part III: Effects of thermal residual stresses. Compos. part A, vol. 38, p. 1581-1596.

Placet V., Trivaudey F., Carbillet S., Mei H., Guicheret-Retel V., Boubakar L. (2011). Potentialités et limites des structures composites à enroulement circonférentiel pour des applications sur machine tournante. $20^{e}$ CFM 2011, Besançon. 
Ratner J.K.H., Chang J.B., Christopher D.A. (2003). Composite flywheel rotor technology - a review. Composite Materials: Testing and Design, vol. 14, p. 3-30.

Starnes J.H., Benson Dexter H., Johnston N.J., Ambur D.R., Cano R.J. (2001). Composite structures and materials research at Nasa Langley research center. Low Cost Composite Structures 2011, Norvège.

Van den Heuvel P.W.J., Van der Bruggen Y.J.W., Peijs T. (1996). Failure phenomena in multi-fibre model composites: Part 1. An experimental investigation into the influence of fibre spacing and fibre-matrix adhesion. Composite part A, vol. 27, p. 855-859. 